\title{
A!
}

This is an electronic reprint of the original article.

This reprint may differ from the original in pagination and typographic detail.

Saarinen, Tapio; Hannula, Jari-Matti; Lehtovuori, Anu; Viikari, Ville

\section{Eight-element MIMO handset based on combinatory feeding}

Published in:

2020 International Workshop on Antenna Technology, iWAT 2020

DOI:

10.1109/iWAT48004.2020.1570599393

Published: 01/02/2020

Document Version

Peer reviewed version

Please cite the original version:

Saarinen, T., Hannula, J-M., Lehtovuori, A., \& Viikari, V. (2020). Eight-element MIMO handset based on combinatory feeding. In 2020 International Workshop on Antenna Technology, iWAT 2020 [9083878] IEEE. https://doi.org/10.1109/iWAT48004.2020.1570599393

This material is protected by copyright and other intellectual property rights, and duplication or sale of all or part of any of the repository collections is not permitted, except that material may be duplicated by you for your research use or educational purposes in electronic or print form. You must obtain permission for any other use. Electronic or print copies may not be offered, whether for sale or otherwise to anyone who is not an authorised user. 


\section{Eight-element MIMO handset based on combinatory feeding}

\author{
Tapio O. Saarinen \\ Department of Electronics and Nanoengineering \\ Aalto University School of Electrical Engineering \\ Espoo, Finland \\ tapio.o.saarinen@aalto.fi
}

\author{
Anu Lehtovuori \\ Department of Electronics and Nanoengineering \\ Aalto University School of Electrical Engineering \\ Espoo, Finland \\ anu.lehtovuori@aalto.fi
}

\author{
Jari-Matti Hannula \\ Department of Electronics and Nanoengineering \\ Aalto University School of Electrical Engineering \\ Espoo, Finland \\ jari-matti.hannula@aalto.fi
}

\author{
Ville Viikari \\ Department of Electronics and Nanoengineering \\ Aalto University School of Electrical Engineering \\ Espoo, Finland \\ ville.viikari@aalto.fi
}

\begin{abstract}
To meet the needs of continuously increasing wireless data consumption, new antenna design schemes are needed. Combinatory feeding is a recently introduced method that utilizes a configurable multiport excitation to create simple frequency configurable antennas. This paper investigates the feasibility of implementing a combinatorially fed antenna capable of $8 \times 8$ MIMO in 1.5-6.5 GHz frequency range and $2 \times 2$ MIMO in $0.7-0.9 \mathrm{GHz}$ frequency range. A prototype antenna is manufactured and measured results demonstrate, that the method is capable of producing antennas of wide bandwidth.
\end{abstract}

Index Terms-Mobile antennas, multifrequency antennas, multiple-input multiple-output (MIMO) antennas, reconfigurable antennas.

\section{INTRODUCTION}

To satisfy the ever so increasing demand for mobile data consumption, multiple approaches are needed. For example, multiple-input-multiple-output (MIMO) techniques can significantly increase the data throughput with the cost of adding antennas to the handset and, thus, reducing the volume available for a single antenna. Devices with $2 \times 2$ and $4 \times 4$ MIMO have been successfully deployed. Yet, the industry is pushing towards higher order systems such as $8 \times 8$ MIMO. Recently, designs up $12 \times 12$ MIMO have been proposed in the literature [1]-[3]. However, most of the proposed designs operate only on one band such as the $3.5 \mathrm{GHz}$ band that will be utilized in $5 \mathrm{G}$ communications.

In addition to the growing number of antennas on a handset, inclusion of multiple new frequency bands in mobile communications places heavy challenges to mobile handset antenna design. Design of traditional multi-resonant or capacitive coupling element (CCE) type antennas is a time consuming task, which typically leads to complicated designs.

As the volume available for the antennas gets sparse and requirement for performance increases, frequency reconfigurable antennas have become an interesting area of research. For example, configurable antennas can utilize tunable aperture matching components or matching circuits to improve the impedance matching over a wider frequency band. However,

This work was supported by Huawei Technologies. in practice, the necessary tuning circuits are complicated and difficult to apply to complex use cases.

A new approach to frequency reconfigurability is the concept of antenna clusters. Multiple promising results have been reported in [4]-[8]. The approach implements frequency reconfigurability by feeding a multiport antenna with differently amplified and delayed signals to minimize the power reflected from the antenna. Instead of separate matching circuits, the method relies on a special multichannel transceiver [6] that is used to weight the signals.

In [9] the concept was extended to a method referred to as combinatory feeding. The frequency response of an antenna cluster can be altered by powering down some of the amplifiers on the multichannel transceiver, which further increases the tuning range of an antenna cluster. Combinatory feeding allows simple antenna elements to be tuned on a wide frequency range.

An antenna utilizing combinatorial feeding has not been implemented by far. In this paper, we study the feasibility of combinatorial feeding in mobile handsets by designing, manufacturing, and measuring a $8 \times 8$ MIMO handset prototype. The proposed handset operates on a wide $1.5-6.5 \mathrm{GHz}$ frequency range with additional of $2 \times 2$ MIMO in the low $0.7-0.9 \mathrm{GHz}$ band.

\section{ANTENNA CLUSTERS}

Combinatory feeding relies on the concept of antenna clusters discussed in [4], [5]. An antenna cluster is an entity consisting of a group of closely spaced antenna elements or a single radiating body with multiple feeds ports. The cluster utilizes the coupling between the tightly spaced elements to create an antenna with optimum performance [4]. Instead of trying to match individual elements or ports to the source impedance, as is done with matching circuits, the approach matches the whole cluster, i.e. to minimize the total power reflected from the cluster. Matching is achieved by feeding each antenna element with a specific amplitude and phase. The 
feeding coefficients can be calculated from the S-parameters of the antenna [10], that are defined as

$$
\mathbf{b}=\mathbf{S a},
$$

where $\mathbf{a}$ and $\mathbf{b}$ are the incident and reflected wave vectors, respectively. The maximum efficiency of an antenna can be simply calculated from the eigenvalues of the scattering matrix $\mathbf{S}$ as

$$
\eta_{\max }=\max \left\{\operatorname{eig}\left(\mathbf{I}-\mathbf{S}^{\mathrm{H}} \mathbf{S}\right)\right\} .
$$

Therefore, the excitation vector a from (1), which provides the best matching efficiency is the eigenvector of $\left(\mathbf{I}-\mathbf{S}^{\mathrm{H}} \mathbf{S}\right)$ that corresponds to the larges eigenvalue $\left(\eta_{\max }\right)$ [5].

\section{COMBINATORY FEEDING}

Idea of combinatory feeding is presented and demonstrated with examples in [9]. By terminating some of the ports with an open circuit, large variety of antenna impedances can be created. By choosing appropriate port combinations, good performance can be obtained over large frequency range.

Instead of feeding the antenna cluster from all ports simultaneously, some of the ports can be left open, which fundamentally changes the frequency response of the cluster. Thus, by going through all possible termination combinations, also referred as to feeding combinations, one cluster with $n$ ports can have up to $n^{2}-1$ different frequency responses, when assuming there are no redundant combinations or symmetry between ports. By increasing the number of ports in a cluster, it is possible to generate enough different frequency responses that all together can cover wide frequency bands. The performance of an antenna cluster is then optimized for a specific frequency band by selecting the most suitable feeding combination.

As the number of ports on the handset increases, the number of all possible combinations increases rapidly. For example, the handset proposed in this paper has 32 ports, which results in approximately $2.56 \times 10^{9}$ combinations at each frequency point. As a result, instead of a brute force approach, an dedicated algorithm is needed for choosing the correct combinations. Development of such optimization algorithm in itself can be a laborious task. Therefore, in this paper, we only utilize a simple algorithm that is described below.

Initially, all ports are active and the algorithm calculates the total efficiencies for all antennas. Next, one feed is turned off, and the worst total efficiencies of the previous and current states are compared. In case the worst efficiency is improved, the respective feed remains in off-state and the same switchoff test is performed to another feed. After multiple tests that do not improve the efficiency of the worst performing antenna, the algorithm stops. After completion, the process is repeated for the next frequency point. To save time, the feed combinations of the previous frequency are used as the starting point for the next frequency.

In practise, the antenna cluster is fed with a special transceiver integrated circuit (IC) which was studied in [7]. The open termination of ports can also be implemented with the same IC. By unbiasing the amplifiers on the transceiver IC, the unbiased amplifier appears as a very small capacitance, i.e. an open circuit, from the perspective of the antenna Currently, no such IC is available for prototyping. Therefore, in this paper, the weighting of excitations is done computationally.

\section{AnTENNA PROTOTYPE}

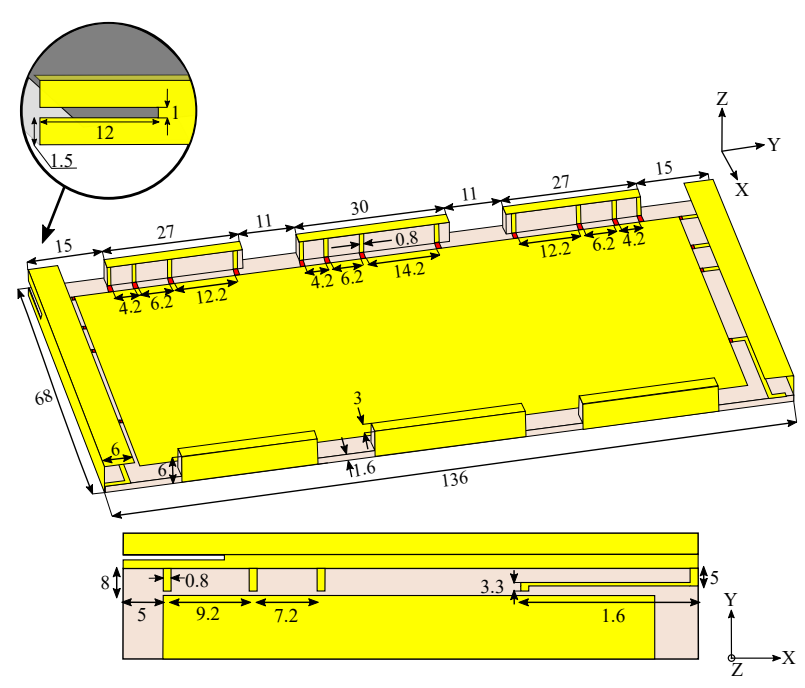

Fig. 1: Dimensions of the manufactured prototype in millimeters.

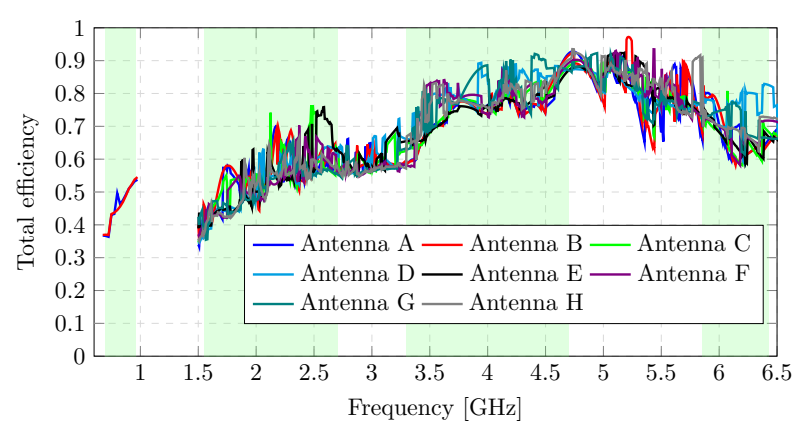

Fig. 2: The simulated total efficiencies of the antennas in $2 \times 2 \mathrm{MIMO}$ in the $0.7-0.9 \mathrm{GHz}$ frequency range and $8 \times 8 \mathrm{MIMO}$ in the $1.5-6.5 \mathrm{GHz}$ range.

\section{A. Design}

To study the concept, we apply the combinatorial feeding method to design and manufacture a $8 \times 8$ mobile handset prototype. The handset has two larger antennas, referred as main antennas, that operate in $0.7-0.9 \mathrm{GHz}$ and $1.5-6.4 \mathrm{GHz}$ frequency ranges. During the design, special emphasis is placed to the following frequency bands: $1.5-2.7 \mathrm{GHz}, 3.3-$ $4.7 \mathrm{GHz}$, and $5.8-6.4 \mathrm{GHz}$. Additionally, the handset has six smaller antennas, referred as data antennas, operating on the same bands from $1.5 \mathrm{GHz}$ onwards. The device is capable of $2 \times 2$ MIMO in the low $0.7-0.9 \mathrm{GHz}$ band and $8 \times 8$ MIMO in the $1.5-6.5 \mathrm{GHz}$ range.

The dimensions of the antenna prototype are illustrated in Fig. 1. The design is based on the $2 \times 2$ handset proposed in [9] with the addition of six higher band data antennas. Each antenna is a folded rectangular sheet with four feed points. The number of feeds was determined by experimentation. Four ports proved sufficient to provide enough combinations 
to cover all the targeted frequency bands. Adding a fifth port was seen unnecessary due to little improvement over increased complexity. The feeds are placed asymmetrically with respect to the antenna element to prevent redundancy of combinations. The specific port locations are fine-tuned by hand to meet targeted frequency bands. The ports and their numbering are illustrated in Fig. 3. The proposed design includes three differently sized antennas: A, C, and D (see Fig. 3). The remaining antennas are merely mirrored to the other side of the handset. For optimal performance, the antenna elements should be placed in the corners of the ground plane [11]. To compensate for the unfavourable location in the lower bands, the data antennas D and G are longer in the y-direction (see Fig. 1 and 3).

Fig. 2 illustrates the simulated efficiencies of the handset in 2 x 2 MIMO in the $0.7-0.9 \mathrm{GHz}$ low-band and 8 x 8 MIMO in the higher bands. In the figure, the efficiencies exhibit sudden jumps and discontinuities. The phenomenon is caused by the selected algorithm as it processes each frequency point individually. In a real application, however, the same coefficients would be used for a wider, for example $20 \mathrm{MHz}$ band. Despite the ripple in the results, the curves give valuable insight about the achievable performance. In MIMO operation, the ergodic capacity is effectively a weighted average of the total efficiencies [12]. As a result, the ripples in individual antennas' responses have only a minor effect to the ergodic capacity.

\section{B. Prototype}

Fig. 3 depicts the manufactured prototype with the antenna and port numbering. The substrate for the handset, including the support blocks for the data antennas, is a single piece of Preperm L260 [13] high frequency plastic. The material has very consistent permittivity and $\tan \delta$ of 0.001 . The antenna elements and groundplane are cut from $0.1 \mathrm{~mm}$ thick bronze and glued to the substrate.

To test the combinatorial feeding without the special IC, two versions of the design are manufactured. One with all the 32 feeds connected and another with the feeds optimized for $4,73 \mathrm{GHz}$. The active ports and weighting coefficients for the single frequency prototype are listed in Table I. The symbol $\infty$ is used to denote an open termination in the respective port.

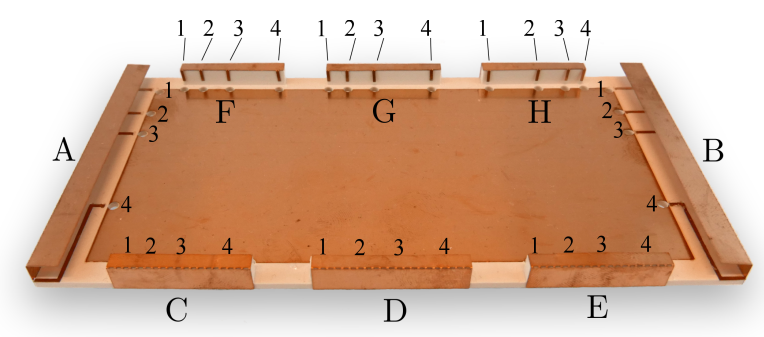

Fig. 3: The manufactured prototype without feed cables.

\section{RESUlTS AND DISCUSSION}

The far-field patterns of the handset are measured with Satimo Starlab antenna measurement system. Fig. 4 illustrates the manufactured prototype with 32 feeds in the measurement

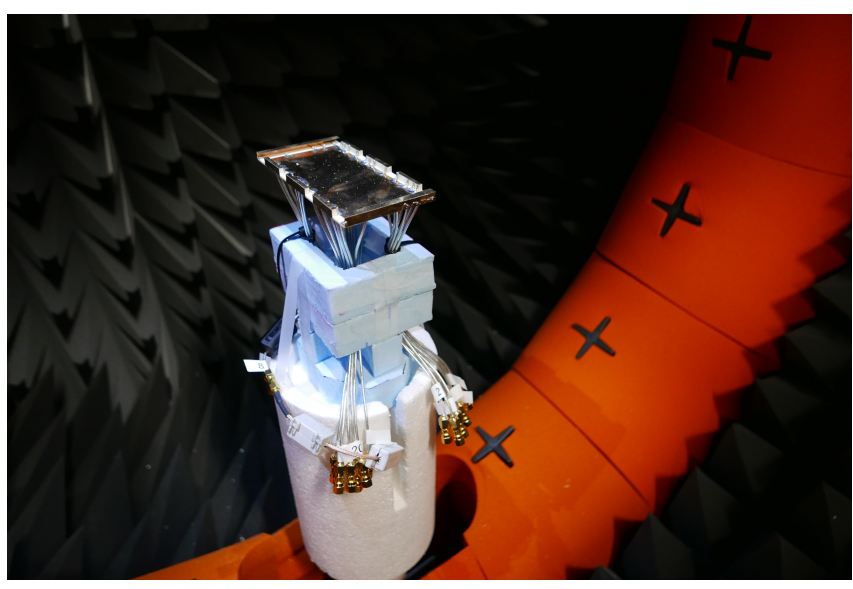

Fig. 4: The 32-port prototype in the Satimo Starlab measurement chamber.

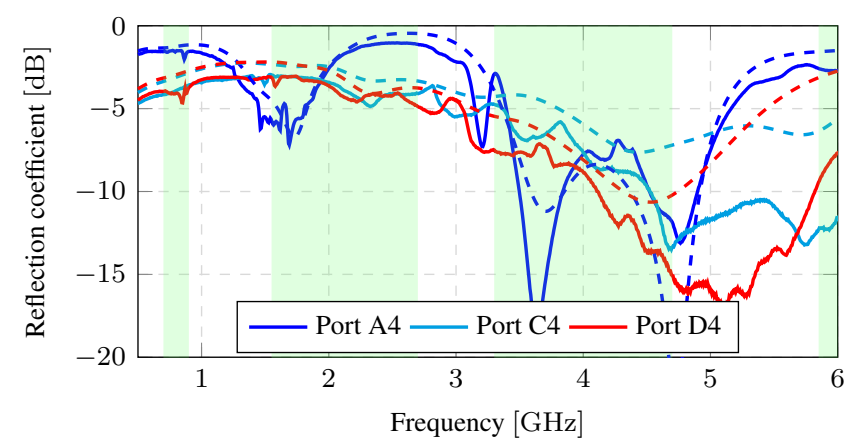

Fig. 5: Three reflection coefficients measured from the full 32-port prototype. Measured results are denoted with solid lines and simulated results are denoted with dashed lines.

chamber. To compute the optimal weighting coefficients for the antennas, the complete S-parameter matrix of the handset is measured with a vector network analyser (VNA). To compare the simulation model and the prototype, three of the measured reflection coefficients are compared to the simulation results in Fig. 5. The three results are selected, as they represent the three different type of antennas on the handset. The measured results are in good agreement with the simulated results, however, due to the feed cable losses, the measured results exhibit consistently lower reflection coefficients.

TABLE I: Optimized feeding coefficients for 8 x 8 MIMO at $4.73 \mathrm{GHz}$

\begin{tabular}{|c|c|c|c|c|}
\hline Antenna & Port 1 & Port 2 & Port 3 & Port 4 \\
\hline A & $0.09 \angle 8.25^{\circ}$ & $\infty$ & $0.47 \angle 2.49^{\circ}$ & $0.88 \angle 0^{\circ}$ \\
B & $0.1 \angle 1.37^{\circ}$ & $\infty$ & $0.46 \angle 0.15^{\circ}$ & $0.88 \angle 0^{\circ}$ \\
C & $\infty$ & $\infty$ & $\infty$ & $1 \angle 0^{\circ}$ \\
D & $0.12 \angle-30.82^{\circ}$ & $0.23 \angle-0.17^{\circ}$ & $0.52 \angle 3.72^{\circ}$ & $0.82 \angle 0^{\circ}$ \\
E & $\infty$ & $\infty$ & $\infty$ & $1 \angle 0^{\circ}$ \\
F & $1 \angle 0^{\circ}$ & $\infty$ & $\infty$ & $\infty$ \\
G & $0.58 \angle-21.45^{\circ}$ & $0.68 \angle-3.20^{\circ}$ & $\infty$ & $0.45 \angle 0^{\circ}$ \\
H & $1 \angle 0^{\circ}$ & $\infty$ & $\infty$ & $\infty$ \\
\hline
\end{tabular}

Although, the results show good correspondence, in further analysis it was observed, that the change in the signal's phase due to the coaxial feeding cables has to be carefully accounted for. The input impedance $Z_{\text {in }}$ of the coaxial cables can be 


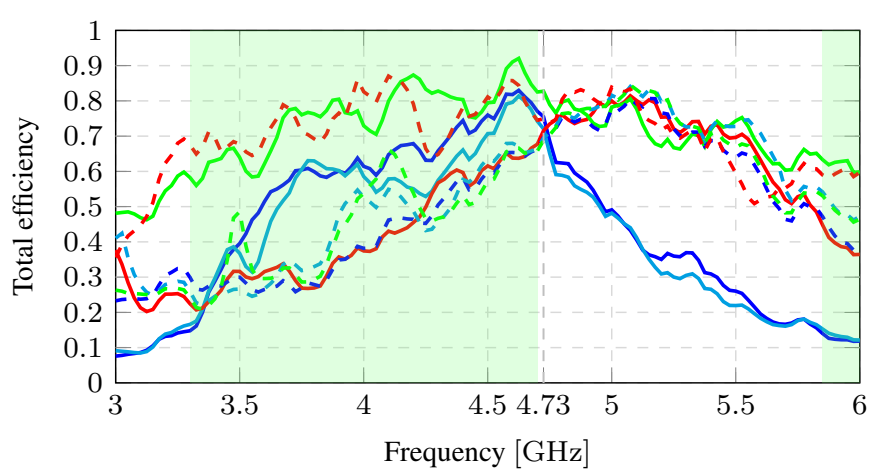

(a)

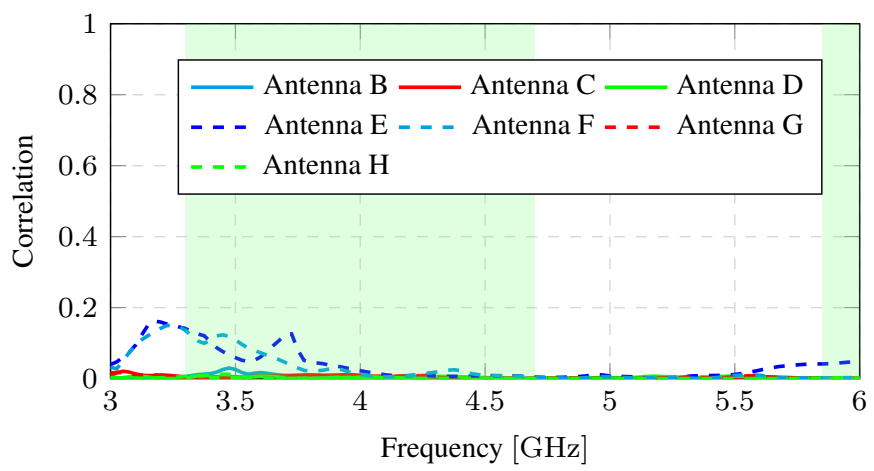

(b)

Fig. 6: (a) Measured efficiencies (b) Measured correlations with respect to antenna $\mathrm{A}$ in $8 \times 8 \mathrm{MIMO}$ with coefficients optimized for $4.73 \mathrm{GHz}$

calculated from the transmission line equation:

$$
Z_{\text {in }}(\ell)=Z_{0} \frac{Z_{\mathrm{L}}+j Z_{0} \tan (\beta \ell)}{Z_{0}+j Z_{\mathrm{L}} \tan (\beta \ell)},
$$

where $Z_{\mathrm{L}}$ is the load impedance, $Z_{0}$ is the characteristic impedance of the cable, $\beta$ is the wavenumber, and $\ell$ is the length of the cable in meters. Due to the periodicity of transmission line impedance, open circuit at the end of the coaxial cable, i.e. $Z_{\mathrm{L}}=\infty$, appears as a periodically changing impedance at the other end. For this reason, the S-parameters measured from the 32-port prototype can not be used to calculate the weighting coefficients for combinatorial excitation. One solution would be to measure the Sparameters of the coaxial cables and use S-parameter based de-embedding methods. However, due to the large number of feed cables, the de-embedding cannot be considered reliable enough. As a result, a dedicated prototype is manufactured and measured to test the simultaneous multiport excitation at 4.73 GHz. Fig. 6a illustrates the measured total efficiencies of the $4.73 \mathrm{GHz}$ prototype. From the figure it can be noticed that the efficiency curves of the eight antennas cross at the targeted frequency. This clearly demonstrates, that the method is able to find coefficients that maximize the total efficiency for all antennas simultaneously.

In practice, the antennas cannot be re-tuned at each frequency. Instead, the antennas must operate over a wider band. The results in Fig. 6a also demonstrate that with a single tuning, all antennas have relatively large $50 \%$ bandwidth for total efficiency. In addition, at the targeted frequency, the correlation between antennas sufficiently low. Fig. 6b illustrates the correlation between antenna elements with respect to antenna A for the $4.73 \mathrm{GHz}$ case.

\section{CONCLUSION}

Applicability of a recently introduced combinatory feeding method has been studied. A handset antenna capable of $2 \times 2$ MIMO in $0.7-0.9 \mathrm{GHz}$ frequency range and $8 \times 8 \mathrm{MIMO}$ in $1.5-6.5 \mathrm{GHz}$ frequency range has been manufactured and measured. The actual utilization of the approach requires a special multi-channel transceiver, nevertheless, the preliminary measurement results demonstrate that the method can be used to produce wideband frequency configurable antennas for high order MIMO handsets.

\section{REFERENCES}

[1] Y. Li, C. Sim, Y. Luo, and G. Yang, "12-port 5G massive MIMO antenna array in Sub-6GHz mobile handset for LTE bands 42/43/46 applications," IEEE Access, vol. 6, pp. 344-354, 2018.

[2] M. Li, Y. Ban, Z. Xu, J. Guo, and Z. Yu, "Tri-polarized 12-antenna MIMO array for future 5G smartphone applications," IEEE Access, vol. 6, pp. 6160-6170, 2018

[3] X. Zhang, Y. Li, W. Wang, and W. Shen, "Ultra-wideband 8-port MIMO antenna array for 5G metal-frame smartphones," IEEE Access, vol. 7, pp. 72 273-72 282, 2019.

[4] J.-M. . Hannula, J. Holopainen, and V. Viikari, "Concept for frequencyreconfigurable antenna based on distributed transceivers," IEEE Antennas and Wireless Propagation Letters, vol. 16, pp. 764-767, 2017.

[5] J.-M. Hannula, T. Saarinen, J. Holopainen, and V. Viikari, "Frequency reconfigurable multiband handset antenna based on a multichannel transceiver," IEEE Transactions on Antennas and Propagation, vol. 65, no. 9, pp. 4452-4460, Sept 2017.

[6] J.-M. Hannula, M. Kosunen, A. Lehtovuori, K. Rasilainen, K. Stadius, J. Ryynanen, and V. Viikari, "Performance analysis of frequency reconfigurable antenna cluster with integrated radio transceivers," IEEE Antennas and Wireless Propagation Letters, 2018.

[7] J.-M. Hannula, T. O. Saarinen, A. Lehtovuori, J. Holopainen, and V. Viikari, "Tunable eight-element MIMO antenna based on the antenna cluster concept," IET Microwaves, Antennas \& Propagation, Feb. 2019.

[8] R. Luomaniemi, J.-M. Hannula, R. Kormilainen, A. Lehtovuori, and V. Viikari, "Unbroken metal rim MIMO antenna utilizing antenna clusters," IEEE Antennas and Wireless Propagation Letters, vol. 18, no. 6, pp. 1071-1075, June 2019.

[9] T. O. Saarinen, J.-M. Hannula, A. Lehtovuori, and V. Viikari, "Combinatory feeding method for mobile applications," IEEE Antennas and Wireless Propagation Letters, vol. 18, no. 7, pp. 1312-1316, July 2019.

[10] C. Volmer, J. Weber, R. Stephan, K. Blau, and M. A. Hein, "An eigen-analysis of compact antenna arrays and its application to port decoupling," IEEE Transactions on Antennas and Propagation, vol. 56, no. 2, pp. 360-370, Feb 2008.

[11] R. Martens, E. Safin, and D. Manteuffel, "Inductive and capacitive excitation of the characteristic modes of small terminals," in Loughborough Antennas Propagation Conference, Loughborough, United Kindom, Nov. 2011, pp. 1-4.

[12] R. Tian, B. K. Lau, and Z. Ying, "Multiplexing efficiency of MIMO antennas," IEEE Antennas and Wireless Propagation Letters, vol. 10, pp. 183-186, 2011.

[13] Premix Oy, “PREPERM®255," Accessed: 27.6.2018. [Online]. Available: https://www.premixgroup.com/products/preperm-255/ 